\title{
Valutazione in vitro dell'associazione di glicopeptidi, ceftazidime e azitromicina nei confronti di Pseudomonas aeruginosa
}

\author{
Barbara Repetto, Pamela Rocher, Anna Marchese, Eugenio A. Debbia. \\ Sezione di Microbiologia - DISCAT, Università di Genova.
}

Parole chiave: Associazione tra antibiotici, glicopeptidi, ceftazidime, azitromicina, Pseudomonas aeruginosa.

\section{SUMMARY}

Objectives: Pseudomonas aeruginosa is an opportunistic human pathogen which is intrinsically resistant to many antibiotics and easily develops resistance towards many currently available agents. Intrisic resistance can be attributed to the low permeability of the $P$. aeruginosa outer membrane to a variety of antibiotics, including glycopeptides (GLYs).

These drugs are active against Gram-positive bacteria and resistance is very rare, it appeared of some interest to evaluate the effect of combining these antimicrobial agents with antibiotics that might disorganize the structure of the outer membrane allowing the entry of glycopeptides into the Gram-negative cells. In order to verify this hypothesis, ceftazidime (CAZ) has been tested in association with vancomycin (VAN) or teicoplanin (TEI). The same experiments have been carried out also in the presence of azithromycin (AZI), which has been shown to interfere with some cellular synthesis in P. aeruginosa.

Methods: A bacterial suspension of about $10^{\circ} \mathrm{CFU} / \mathrm{ml}$ was seeded on plates containing a fixed concentration of GLYs $(500 \mathrm{mg} / \mathrm{l})$ and increasing doses $(2 x, 4 x, 8 x, 16 x)$ of CAZ. Survivors were counted after 48 hs at $37^{\circ} \mathrm{C}$. Results were interpreted as synergism $(99 \%)$, additivity $(90 \%)$, and indifference $(10 \%)$ of the CFU/ml reduction found in the drugs combination in comparison to the drug alone. The same experiments have been repeated adding AZI ( $16 \mathrm{mg} / \mathrm{l})$ and using GLYs at concentrations ranging from 500 to $300 \mathrm{mg} / \mathrm{l}$.

Results: CAZ in combination with GLYs reacted synergically in 20 out of 59 cases, additivity was found in $31 / 59$ interactions and indifference was noted in $8 / 59$ tests. Preliminary results ( 12 tests performed) indicated that the addition of $A Z I$ increased the incidence of synergisms and additivities even when using GLYs concentration of $300 \mathrm{mg} / \mathrm{l}$ (figure I).

Conclusions: CAZ combined with GLYs gave additive or synergistic results in the geat majority of experiments, while the simultaneous combination of AZI, CAZ and a GLY increased the incidence of additive or synergistic effect against $P$. aeruginosa. These data, given the high concentration of GLYs employed, could be of particular interest in clinical situations where the drugs could be topically administered.

\section{INTRODUZIONE}

Pseudomonas aeruginosa è un microrganismo ubiquitario che in virtù delle sue versatili proprietà biochimiche può comportarsi da patogeno opportunista causando infezioni gravi e spesso letali in pazienti immunocompromessi. Esso rappresenta di fatto il più frequente germe isolato da infezioni ospedaliere essendo responsabile del 10$20 \%$ circa delle infezioni nosocomiali $(3,7,8)$.

$P$. aeruginosa è intrinsecamente resistente a molte classi di farmaci (penicilline, cefalosporine di prima e seconda generazione, tetracicline, macrolidi, cloramfenicolo, sulfamidici) i quali non riescono a penetrare all'interno della cellula batterica; tale germe inoltre evolve frequentemente verso una multiresistenza nei confronti di agenti inizialmente attivi. A tale riguardo questo studio si è proposto di indagare il possibile utilizzo dei glicopeptidi nel trattamento delle infezioni sostenute da $P$. aeruginosa: è infatti noto che l'evolversi di una resistenza spontanea nei confronti di tali sostanze antibatteriche è quasi impossibile poiché, perché questo accada, sono necessari tre distinti eventi mutazionali. I glicopeptidi, tuttavia, non sono attivi sui Gram-negativi a causa del lipopolisaccaride (LPS) che ne impedisce l'ingresso. Per aggirare questo ostacolo ci si è rifatti al caso degli Enterococchi, ove gli aminoglicosidi non riescono ad entrare spontaneamente, ma solo in associazione con l'ampicillina che ha il compito di creare dei "varchi" sulla parete batterica $(12,16)$. Allo stesso modo si è pensato di poter utilizzare sostanze come il ceftazidime o la tobramicina per disorganizzare la struttura del lipopolisaccaride e creare dei "varchi" sulla parete di $P$. aeruginosa così da permettere il passaggio dei glicopeptidi all'interno della cellula batterica $(12,16)$. La sperimentazione non ha tenuto conto della concentrazione raggiungibile in vivo dai glicopeptidi, ma solo di verificare se esiste un'interazione favorevole tra questi farmaci, indipendentemente dalla dose utilizzata, per contrastare la 
proliferazione di questo patogeno opportunista. Lo studio ha valutato, in una prima fase, l'attività di ceftazidime e tobramicina in combinazione con vancomicina su isolati clinici di $P$. aeruginosa. È stata quindi esplorata l'efficacia di teicoplanina in sostituzione del primo glicopeptide. Tali combinazioni di antibiotici sono state infine valutate alla presenza di azitromicina che, pur non essendo normalmente attiva su $P$. aeruginosa, ha dimostrato, alle concentrazioni raggiungibili in vivo, di poter interferire sulla produzione di diversi metaboliti in questo patogeno $(1,4,5,6,9,11$, 14,15,17).

\section{MATERIALI E METODI}

\section{CEPPI BATTERICI}

Sono stati utilizzati in totale $59 P$. aeruginosa provenienti da bronco aspirati, espettorati, emocolture e tamponi ferita di pazienti diversi ricoverati in reparti a rischio e isolati nel laboratorio di Microbiologia Clinica dell'Università degli Studi di Genova. I ceppi sono stati identificati secondo procedure standard (8).

\section{SAGGIO DI SENSIBILITÀ}

Ceftazidime, tobramicina, vancomicina, teicoplanina e azitromicina utilizzati in questa sperimentazione sono stati ottenuti direttamente dal produttore o acquistati in commercio. La soluzione madre $(10 \mathrm{mg} / \mathrm{l})$ è stata allestita secondo le istruzioni del fornitore o come suggerito da Clinical Laboratory Standards Institute (CLSI) (in precedenza, NCCLS) (2) e diluita nel terreno prescelto. La determinazione della minima concentrazione inibente è stata eseguita secondo il metodo della microdiluizione in Mueller Hinton (MH) brodo e i microrganismi sono stati classificati come sensibili, intermedi e resistenti secondo le linee guida del CLSI (2). P. aeruginosa ATCC 27853 è stato utilizzato come ceppo di controllo.

\section{SELEZIONE DI STIPITI RESISTENTI}

I ceppi sono stati coltivati a $37^{\circ} \mathrm{C}$ in brodo fino ad una concentrazione di almeno $10^{\circ} \mathrm{FU} / \mathrm{ml}$ e poi seminati su piastre contenenti una concentrazione fissa inziale di $500 \mathrm{mg} / \mathrm{l}$ di vancomicina o teicoplanina e diluizioni scalari $(2 \mathrm{x}, 4 \mathrm{x}, 8 \mathrm{x}, 16 \mathrm{x})$ di ceftazidime o tobramicina a partire $4 \mathrm{mg} / \mathrm{l}$. La concentrazione dei glicopeptidi è stata ridotta a $300 \mathrm{mg} / 1$ negli esperimenti eseguiti in presenza di azitromicina.

Dopo una incubazione di 48 ore a $37^{\circ} \mathrm{C}$ sono stati contati i sopravvissuti e i risultati sono stati interpretati come sinergismo $(99 \%)$, additività $(90 \%)$ o indifferenza $(0-10 \%)$ sulla base della riduzione delle $\mathrm{CFU} / \mathrm{ml}$ ritrovate con gli antibiotici in com- binazione rispetto al singolo composto. Tale criterio, pur essendo arbitario, si rifà alle norme che interpretano i risultati delle interazioni tra antibiotici adottate nei saggi di batteriocidia dinamicica mediante il disegno delle curve che riportano il numero dei batteri sopravvissuti in funzione del tempo (13).

\section{RISULTATI \\ Interazioni tra ceftazidime e tobramicina con vancomicina e teicoplanina.}

Gli esperimenti preliminari intesi a valutare il possibile utilizzo dei glicopeptidi in associazione con ceftazidime o tobramicina nel trattamento delle infezioni sostenute da $P$. aeruginosa sono stati condotti su 53 ceppi risultati resistenti ad uno $o$ più farmaci. Tali germi sono stati seminati su piastre contenenti una concentrazione costante di vancomicina $(500 \mathrm{mg} / \mathrm{l})$ e diluizioni scalari di ceftazidime. Tale concentrazione è stata scelta dopo una valutazione preliminare su un numero limitato di ceppi stimando concentrazioni scalari di vancomicina $(2000,1500,1000,750,500$ e 250 $\mathrm{mg} / \mathrm{l})$ in associazione con il ceftazidime.

Il ceftazidime in combinazione con vancomicina ha reagito in modo sinergico in 19/53 casi, additività è stata trovata in 24/53 interazioni e 10 saggi su 53 hanno mostrato indifferenza. In nessun caso il ceftazidime in associazione con vancomicina ha reagito in modo antagonista (tabella 1).

Successivamente alla vancomicina è stata aggiunta la tobramicina ed è stato registrato sinergismo in 10/53 casi, additività in 20/53 casi e le rimanenti 23 interazioni hanno mostrato una reazione indifferente. Anche in questo caso non sono stati registrati casi di antagonismo (tabella 1).

Successivamente gli esperimenti sono stati ripetuti sostituendo alla vancomicina la teicoplanina, altro glicopeptide che sembra avere il vantaggio di essere maggiormente attivo nei confronti dei Gram-negativi. Contemporaneamente è stata diminuita la concentrazione dei glicopeptidi (400 $\mathrm{mg} / \mathrm{l})$. I risultati eseguiti su metà dei ceppi scelti a caso hanno evidenziato una sovrapposizione tra $\mathrm{i}$ dati conseguiti con vancomicina e quelli osservati con teicoplanina.

Il ceftazidime in associazione con i glicopeptidi ha reagito in modo sinergico in 20/59 casi, additività è stata riscontrata in $31 / 59$ interazioni mentre i restanti 8 casi hanno mostrato indifferenza (tabella 2). Alla luce di questi risultati, la tobramicina non è stata ulteriormente saggiata.

Interazioni tra ceftazidime, glicopeptidi e azitromicina.

Per incrementare il numero degli effetti sinergici ottenuti nelle prime fasi dello studio è stata com- 
binata al ceftazidime e ai glicopeptidi l'azitromicina che, come già detto, pur non essendo attiva su $P$. aeruginosa ha dimostrato di poter interferire con diverse sintesi di questo microrganismo. In particolare è stato ipotizzato che l'aggiunta di azitromicina potesse impedire al germe di "tamponare" i danni causati dal ceftazidime sulla parete batterica incrementando l'effetto sinergico dell'associazione tra gli antibiotici.

Gli esperimenti eseguiti su 12 ceppi hanno in effetti mostrato come l'aggiunta di azitromicina $(16 \mathrm{mg} / \mathrm{l})$ incrementi l'incidenza di sinergismi e additività quando $\mathrm{i}$ glicopeptidi sono utilizzati ad una concentrazione di $300 \mathrm{mg} / \mathrm{l}$. Scegliendo infat- ti arbitrariamente 12 ceppi che avevano mostrato precedentemente di reagire 4 in modo sinergico, 4 dando additività e 4 mostrando indifferenza, è stato osservato un incremento di una unità nel numero dei sinergismi e di 2 unità nel numero delle additività con conseguente diminuzione del numero di indifferenze (tabella 3 ).

La riduzione delle CFU $/ \mathrm{ml}$ di $P$. aeruginosa trattato con ceftazidime in combinazione con i glicopeptidi $300 \mathrm{mg} / \mathrm{l}$ e l'azitromicina $16 \mathrm{mg} / \mathrm{l}$, rispetto alle $\mathrm{CFU} / \mathrm{ml}$ riscontrate nel caso di trattamento con solo ceftazidime e glicopeptidi, è evidenziata nella figura I.

Tabella I. Interazione tra ceftazidime e tobramicina con vancomicina $(500 \mathrm{mg} / \mathrm{l})$ nei confronti di 53 diversi ceppi di P. aeruginosa

\begin{tabular}{ccccc}
\hline ANTIBIOTICI & $N^{\circ}$ & SINERGISMO & ADDITIVITÀ & INDIFFERENZA \\
CAZ+VAN & 53 & 19 & 24 & 10 \\
\hline TOB+VAN & 53 & 10 & 20 & 23 \\
\hline
\end{tabular}

CAZ, ceftazidime; TOB, tobramicina; $\mathrm{VAN}$, vancomicina.

Tabella 2. Interazione tra ceftazidime e i glicopeptidi vancomicina o teicoplanina (400 mg/l) nei confronti di 59 diversi ceppi di P. aeruginosa

\begin{tabular}{ccccc}
\hline ANTIBIOTICI & $\mathrm{N}^{\circ}$ & SINERGISMO & ADDITIVITA' & INDIFFERENZA \\
\hline CAZ+GLI & 59 & 20 & 31 & 8
\end{tabular}

CAZ, ceftazidime; GLI, glicopeptidi.

Tabella 3. Interazione tra ceftazidime e i glicopeptidi vancomicina o teicoplanina (300 mg/l) nei confronti di 12 diversi ceppi di P. aeruginosa in presenza di azitromicina ( $16 \mathrm{mg} / \mathrm{l})$

\begin{tabular}{ccccc}
\hline ANTIBIOTICI & $N^{\circ}$ & SINERGISMO & ADDITIVITA' & INDIFFERENZA \\
\hline CAZ+GLI & 12 & 4 & 4 & 4 \\
\hline CAZ+GLI +AZI & 12 & 5 & 6 & I
\end{tabular}

CAZ, ceftazidime; GLI, glicopeptidi;AZI, azitromicina.

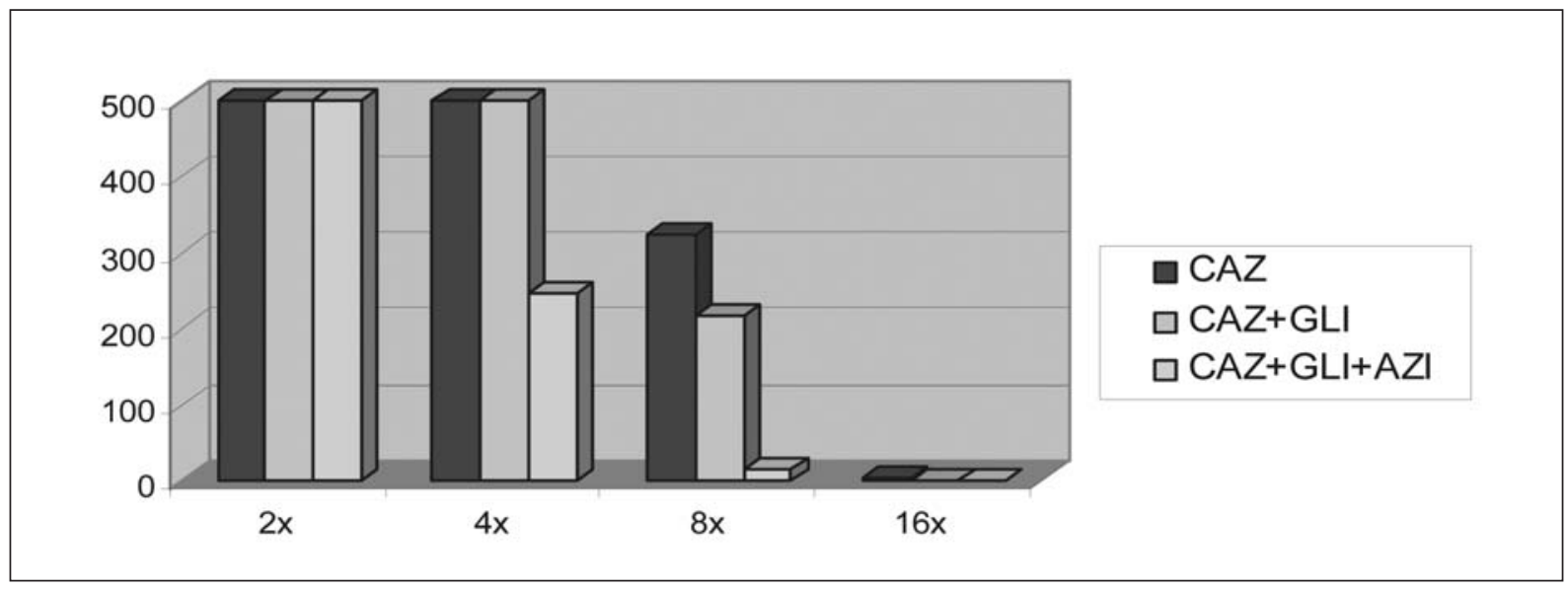

Figura I. CFU/ml di P. aeruginosa coltivato su piastre contenenti ceftazidime (CAZ) da solo e in combinazione con i glicopeptidi (GLI) $300 \mathrm{mg} / \mathrm{l}$ e l'azitromicina (AZI) $16 \mathrm{mg} / \mathrm{l}$.

I valori delle $\mathrm{CFU} / \mathrm{ml}$ sono stati normalizzati sulla base di un massimo pari a 500 


\section{CONCLUSIONI}

I risultati ottenuti indicano una favorevole interazione fra il ceftazidime o la tobramicina con i glicopeptidi vancomicina e teicoplanina. In nessun caso l'associazione ha evidenziato effetti di antagonismo. La combinazione degli antibiotici ha dimostrto inoltre di beneficiare dell'apporto dell'azitromicina sia in termini di interazioni sinergiche che in termini di concentrazione di farmaci utilizzati.

Anche se questi studi richiedono ulteriori prove a conferma, vale la pena di sottolineare che antibiotici come i glicopeptidi, se utilizzati in terapia nelle infezioni sostenute da $P$. aeruginosa potrebbero suggerire un approccio terapeutico innovativo, specie per le situazioni più gravi come la fibrosi cistica.

L'eventuale utilizzo dei glicopeptidi o di farmaci della stessa classe, fornirebbe inoltre un margine temporale piuttosto lungo, se si considera che la vancomicina è in terapia da oltre 40 anni e che $\mathrm{i}$ primi veri resistenti in $S$. aureus sono emersi soltanto di recente (10).

Il rovescio della medaglia è rappresentato da un possibile incremento dell'uso di questi principi attivi che potrebbe accelerare l'evoluzione negativa già in atto nei Gram-positivi.

Sono aspetti che vanno tenuti presenti, ma nello stesso tempo è importante conoscere sempre più a fondo l'interazione fra queste molecole attraverso studi delle curve di batteriocidia per comprendere se gli effetti letali osservati sono di tipo batteriostatico o battericida e per capire quali sarebbero eventualmente i dosaggi più adatti da utilizzare in terapia, specie nei casi in cui i farmaci possono essere somministrati per via topica, come nel caso citato della fibrosi cistica.

\section{BIBLIOGRAFIA}

1. Altschuler EL. Azithromycin, the multidrug-resistant protein and cystic fibrosis. Lancet, 1998; 351: 1286.

2. Clinical and Laboratory Standards Institute. Performance Standards for Antimicrobial Susceptibility Testing; Fifteenth Informational Supplement M100 S15 (2005). Wayne, PA.

3. Durack,DT. Pseudomonas aeruginosa: a ubiquitous pathogen. In Schaechtcr M, Medoff G, Schlessinger D. Mechanisms of microbial disease. William \& Wilkins, Baltimore 1989

4. Giamarellou, H. Prescribing guidelines for severe Pseudomonas infections. J Antimicrob Chemoter 2002; 49: 229-33.

5. Høiby N. New antimicrobials in the management of cystic fibrosis. J Antimicrob Chemother 2002; 49: 235-8.

6. Howe RA, Spencer RC. Macrolides for the treatment of Pseudomonas aeruginosa infections J Antimicrob Chemother 1997; 40: 153-5.
7. Hutchinson ML, Govan JRW. Pathogenicity of microbes associated with cystic fibosis. Microbes Infect 1999; 1: 1005-14.

8. Kiska DL, Gilligan PH. Pseudomonas. In Murray PR, Baron EJ, Pfaller MA, Tenover FC, Yolken RH. Manual of Clinical Microbiology. 7th ed. American Society for Microbiology Press Washington, DC, 2003; 517-26.

9. Lyczak JB, Cannon CL, Pier GB. Lung infections associated with cystic fibrosis. Clin Microbiol Rev 2002; 15: 194-222.

10. Miller DV, Urdaneta, Weltman A. Vancomycin-resistant Staphylococcus aureus - Pennsylvania 2002. JAMA 2002; 288: 2116.

11. Molinari G, Guzman C, Pesce A, Schito GC. Inhibition of Pseudomonas aeruginosa virulence factors by sub-inhibitory concentrations of azithromycin and other macrolides antibiotics. J Antimicrob Chemother 1993; 31: 681-8.

12. Nikaido H. Molecular basis of bacterial outer membrane permeability revisited. Microbiol Rev 2003; 67: 593-656.

13. Pillai SK, Moellering RC, Eliopoulos GM. Antimicrobial combinations. In: Lorian V, Antibiotics in Laboratory Medicine, Fifth Ed. Lippincott, Williams and Wilkinson, 2005; 352-440.

14. Shryock TR, Mortensen JE, Baumholtz M. The effects of macrolides on the expression of bacterial virulence mechanisms. J Antimicrob Chemother 1998; 41: 505-12.

15. Tateda K, Comte R, Pechere JC, Kohler T, Yamaguchi $\mathrm{K}$, VanDelden C. Azithromycin inhibits quorum sensing in Pseudomonas aeruginosa. Antimicrob Agents Chemother 2001; 45: 1930-3.

16. Vaara M. Agents that increase the permeability of the outer membrane. Microbiol Rev 1992; 56: 395-411.

17. Yanagihara K, Tomono K, Sawai T, Kuroki M, et al. Combination therapy for chronic Pseudomonas aeruginosa respiratory infection associated with biofilm formation. 2000; 46: 69-72.
Eugenio A. Debbia

Università degli Studi di Genova

DISCAT - Sezione di Microbiologia

Largo Rosanna Benzi 10

6132 Genova - Italia

Tel. ++010-3537655; Fax ++010-3537698

E-mail: eugenio.debbia@unige.it 